\title{
Analyse rétrospective de la croissance radiale et mise en relation avec le bilan hydrique dans un dispositif d'intensité d'éclaircie de pin maritime dans les Landes de Gascogne
}

\author{
Jean Timbal \\ Unité INRA de Recherches Forestières, Équipe Écophysiologie et Nutrition, Domaine de l'Hermitage, \\ Pierroton, 33610 Cestas, France
}

(Reçu le 31 mai 2001 ; accepté le 7 novembre 2001)

\begin{abstract}
Résumé - En utilisant la technique dendrochronologique, une analyse rétrospective de la croissance radiale a été effectuée en 1998 sur un dispositif sylvicole de pin maritime (Pinus pinaster) des Landes de Gascogne destiné à étudier l'influence sur la croissance de différents régimes d'éclaircie. Ce dispositif installé en 1967 dans un peuplement de 19 ans, ayant actuellement 52 ans, avait été suivi pendant 12 ans. Il comprend 5 modalités d'intensité d'éclaircie avec 5 répétitions. Cette étude dendroécologique a permis de comparer l'effet des différentes intensités d'éclaircies testées et d'analyser les interactions, dans le contexte pédo-climatique landais, entre l'effet sur la croissance radiale des éclaircies et celui des circonstances climatiques dans lesquelles s'est déroulée cette croissance, essentiellement l'effet des sécheresses estimées par le bilan hydrique calculé pour la saison de croissance correspondante. D’une manière logique, les résultats acquis ont montré que des éclaircies fortes permettaient bien sûr une meilleure croissance radiale mais aussi une meilleure résistance des arbres au stress hydrique « ordinaire ». En revanche, les années de forte sécheresse, ces fortes éclaircies n'ont pas suffi à empêcher une réduction sensible de la croissance radiale ; réduction d'autant plus importante que l'intensité des éclaircies est forte. De plus, pour la tranche d'âge étudiée (20 à 35 ans) l'effet d'une éclaircie ne se manifeste que durant 4 à 5 ans. Sur le plan pratique, ces résultats confirment l'intérêt d'éclaircies fréquentes et d'intensité assez forte compatible cependant, sur le plan économique, avec le maintien sur pied d'un nombre de tige, et donc d'un volume, suffisant.
\end{abstract}

dendroécologie / croissance radiale / Pinus pinaster / intensité d'éclaircie / bilan hydrique

\begin{abstract}
Retrospective analysis of radial growth in a Pinus pinaster thinning experiment in the Landes de Gascogne. Relationship with the water balance. A dendrochronological analysis was carried out in 1998, in a Pinus pinaster thinning experiment in SW France. This experiment was established in 1967 (at 19 years old). It consists on 5 thinning intensities with 5 repetitions. Radial growth pattern in each thinning modality has been compared and annual interactions between radial growth and water balance - from Thornthwaite ETP formula - have been studied in the Landes soils and climate environment. At high thinning intensity, trees display higher water stress resistance. Nevertheless, during the years with very low rainfall, radial growth is much reduced in high thinning intensity plots, which is a mesophytic trait. During this thinning experiment, with tree age ranking from 20 to 35 , thinning effect duration was no longer than 4-5 years. On a sylvicultural view-point, these results point out the interest of frequent thinning practices (every 4 or 5 years) with an economical equilibrium between high thinning intensity and stand density.
\end{abstract}

dendroecology / radial growth / Pinus pinaster / thinning intensity / water balance

Correspondance et tirés-à-part

Tél. 0557122300 ; Fax. 0556680546 ; e-mail : timbal@pierroton.inra.fr 


\section{INTRODUCTION}

Les éclaircies constituent une opération essentielle de la pratique sylvicole [20]. Ainsi, dans les futaies régulières de feuillus (hêtre ou chênes), ces éclaircies sont généralement régies par une "norme », c'est-à-dire par une relation mathématique reliant l'âge (ou la hauteur dominante, ou la circonférence, mieux accessibles) à la densité du peuplement. Dans les forêts cultivées d'une manière intensive, en particulier celle, monospécifique, de pin maritime (Pinus pinaster) des Landes de Gascogne, le régime des éclaircies constitue une composante très importante des itinéraires techniques préconisés. On trouve une telle norme dans l'ouvrage de J.P. Maugé [18] sur la sylviculture du pin maritime dans les Landes de Gascogne. Cette norme se présente sous forme d'un tableau indiquant, pour chaque circonférence, de 50 à $95 \mathrm{~cm}$, une densité maximale et une densité minimale préconisées. La table de production de Lemoine et Decourt [16] est plus précise puisqu'elle donne, pour chaque âge, et pour les divers niveaux de fertilité du sol (site index), après éclaircie, ce que devraient être les diverses caractéristiques dendrométriques du peuplement, y compris le volume et l'accroissement courant.

Pour établir scientifiquement ces itinéraires techniques de la culture du pin maritime, une expérimentation a été entreprise en 1966 consistant à suivre régulièrement la croissance sur des placettes soumises à des intensités variables d'éclaircie. Les résultats de ces mesures dendrométriques classiques ont fait l'objet de plusieurs publications : Illy et Lemoine [10], Lemoine [14], Lemoine et Sartolou [17].

Trente ans après la mise en place de cette expérimentation, au moment où le peuplement est parvenu en fin de révolution et doit passer prochainement en coupe rase, il était intéressant de procéder, dans les divers placeaux, à une analyse rétrospective de la croissance radiale sur carottes de sondage, afin d'analyser et de comparer plus finement la dynamique de croissance radiale dans les différentes intensités d'éclaircie et d'essayer de démêler l'action de ces éclaircies de celle des circonstances climatiques. C'est le résultat de l'analyse dendroécologique que nous présentons ici.

Si l'effet des éclaircies dans des peuplements réguliers, en particulier de pins, a donné lieu a de nombreuses études écophysiologiques (en particulier Donner et Running [6], Ginn et al. [8], Law et al. [11]) ou sur la croissance globale des peuplements forestiers, aussi bien feuillus que résineux, jeunes ou vieux, naturels ou issus de plantation, peu nombreuses sont celles qui ont abordé ces questions par l'approche dendrochronologique. Pour certaines, la problématique n'était pas l'effet des éclaircies en soi, mais, plus fondamentalement, l'influence sur les processus physiologiques de la densité des peuplements (Badeau et al. [1]) et la compétition entre les individus à l'intérieur des peuplements (Becker [3]). Une mention particulière doit être faite de l'étude de Le Goff et Ottorini [13] qui, comme nous-même, ont étudié à l'aide d'une technique dendrochronologique, sur Fagus sylvatica, un dispositif ancien d'éclaircies installé en Lorraine. Signalons aussi l'étude de Cutter et al. [5] sur un peuplement mixte de chênes (Quercus coccinea et Q. velutina) du Missouri.

Très nombreuses sont les études portant sur les relations entre croissance radiale et les fluctuations climatiques inter annuelles, aussi bien sur des espèces feuillues que résineuses, et cela dans des pays et donc des climats très divers. Citons quelques unes des plus récentes : Celle de Splechtna et al. [23] sur un sapin à l'étage subalpin des Montagnes Rocheuses, celle de Parker et al. [21] sur deux variétés de Pinus clausa en Floride et celle de Rozas [22] sur Quercus robur et Fagus sylvatica dans la Cordillère cantabrique (Nord Espagne). Certains, comme Lebourgeois [12] sur Pinus laricio dans l'Ouest de la France, se sont même attachés à analyser les relations entre facteurs climatiques et croissance des bois de printemps et d'été.

L'étude dendroécologique la plus récente sur le thème des interactions entre climat, éclaircies et croissance radiale sur résineux est certainement celle de Misson [19] portant sur 3 plantations de Picea abies de Wallonie (Belgique) et dans laquelle cet auteur a cherché a faire la part de l'effet des éclaircies proprement dites de celui des effets climatiques concomitants.

Signalons enfin l'étude plus générale de Lemoine [15] sur l'effet des sécheresses sur la croissance du pin maritime dans les Landes de Gascogne.

\section{MATÉRIEL ET MÉTHODES}

\subsection{Le site expérimental}

L'expérimentation a été implantée en 1966 dans une parcelle du domaine INRA de l'Hermitage à Cestas (33). L'altitude y est de $58 \mathrm{~m}$ et la topographie est plate.

Il s'agit d'une parcelle de 7 ha couverte d'une futaie régulière de pins maritimes issue de semis en bandes (labourées mais non fertilisées) réalisés en 1947 après un 
incendie. Les bandes avaient une largeur de $2 \mathrm{~m}$ et les inter-bandes de $3 \mathrm{~m}$.

Sur le plan écologique, la parcelle est relativement homogène. En effet, il s'agit d'une lande humide à Molinia caerulea dominant, passant en quelques endroits à une lande plus mésophile à Pteridium aquilinum ; ces variations de végétation spontanée du sous-bois sont liées aux variations du régime de fluctuation de la nappe présente à faible profondeur dans le sol. Ce dernier se classe dans la catégorie des podzols humiques hydromorphes à alios. Ces conditions sont bien représentatives d'une grande partie du massif de pin maritime des Landes de Gascogne.

\subsection{Le dispositif expérimental}

Le dispositif a été installé sur cette parcelle en 1966-1967. Le peuplement de pins maritimes avait alors 19 ans et avait déjà été soumis à plusieurs éclaircies qui, au vu de la densité des témoins, avaient été sans doute d'assez faible intensité. Le peuplement correspondait, d'un point de vue productivité, à ce qui a été défini peu après comme la classe II de la table de production de Lemoine et Decourt [16].

Le dispositif consiste en 5 blocs plus ou moins éclatés de 5 placeaux chacun; placeaux correspondant aux 5 modalités et répartis au hasard à l'intérieur des blocs. Ces blocs avaient été assis en fonction des caractéristiques dendrométriques du peuplement sensées refléter les variations de fertilité du sol (Lemoine [14]). Il n'y a pas d'interaction entre blocs et traitements.

Les 5 modalités d'éclaircie sont les suivantes : $1(=\mathrm{T})$ : témoin ; 2 : éclaircie faible ; 3 : éclaircie moyenne ; 4 : éclaircie forte ; 5 : éclaircie très forte.

Pour les «témoins» il n'a été pratiquée aucune éclaircie systématique, seulement les coupes sanitaires indispensables.

Chaque type d'éclaircie est caractérisé par une surface terrière et un facteur d'espacement [17].

Il y a 4 répétitions, ce qui fait un total de $5 \times 5=25$ placeaux. La surface de chacun de ces placeaux n'est pas constante ; elle varie de 10 à 15 ares en fonction de l'intensité d'éclaircie pratiquée.

Cette expérimentation a duré 12 ans, de 1967 à 1979, c'est-à-dire jusqu'à 32 ans, durant lesquels 4 éclaircies, espacées de 4 puis 5 ans, ont été réalisées. Au cours de ces éclaircies, on a cherché à maintenir autant que possible les densités initiales fixées pour l'expérimentation.
Les surfaces terrières correspondantes sont données dans le tableau I tiré de Lemoine et Sartolou [17] et complété.

Les 4 éclaircies ont été réalisées : en 1966 (au moment de l'installation du dispositif, à 19 ans), en 1970 (à 23 ans), en 1974 (à 27 ans) et en 1979 (à 32 ans).

Remarquons que dire qu'une éclaircie à été réalisée à l'année $n$ veut dire implicitement qu'elle a été effectivement réalisée après la saison de végétation de cette année, c'est-à-dire à l' automne de l'année $n$, voire durant l'hiver de l'année $n-(n+1)$.

Au moment de chaque éclaircie, deux inventaires dendrométriques (avant et après) ont été réalisés. En 1998, avant le prélèvement des carottes de sondage, un inventaire total des circonférences à été réalisé sur tous les placeaux. Le résultat de cet inventaire (circonférences et densités de peuplement) est donné, sous forme de diagramme, par la figure 1 .

\subsection{L'échantillonnage}

Un prélèvement de carottes de sondage a été effectué en juillet 1998. Pour chaque arbre échantillonné, il a été prélevé, à l'aide de tarières de Pressler de $40 \mathrm{~cm}$ de long et de $5 \mathrm{~mm}$ de diamètre intérieur, à la base du tronc, juste au-dessus de l'empattement, et à même azimut (au sud) une seule carotte à cœur.

Dans les 25 placeaux, une trentaine d'arbres (de 26 à 32) a ainsi été sondée. Ce nombre représente la totalité des arbres dans les modalités de forte et très forte éclaircie et seulement un échantillon dans les modalités témoin ou à faible éclaircie ; échantillon réalisé sur la base de l'inventaire des circonférences fait préalablement, avec sélection

Tableau I. Surfaces terrières $\left(\mathrm{m}^{2} \mathrm{ha}^{-1}\right)$ relatives (par rapport aux témoins) obtenues dans les différentes modalités d'éclaircie, après chaque éclaircie réalisée au cours de l'expérimentation.

Rotation entre 1966-1970 1970-1974 1974-1979 1979-1985 les éclaircies

\begin{tabular}{lcccc} 
Âge au début & 19 ans & 23 ans & 27 ans & 32 ans \\
\hline Témoin & 100 & 100 & 100 & 100 \\
$\begin{array}{l}\text { Éclaircie faible } \\
\text { Éclaircie }\end{array}$ & 85 & 80 & 78 & 82 \\
$\begin{array}{l}\text { moyenne } \\
\text { Éclaircie forte }\end{array}$ & 68 & 70 & 66 & 70 \\
$\begin{array}{l}\text { Éclaircie très } \\
\text { forte }\end{array}$ & 60 & 52 & 51 & 50 \\
\hline
\end{tabular}




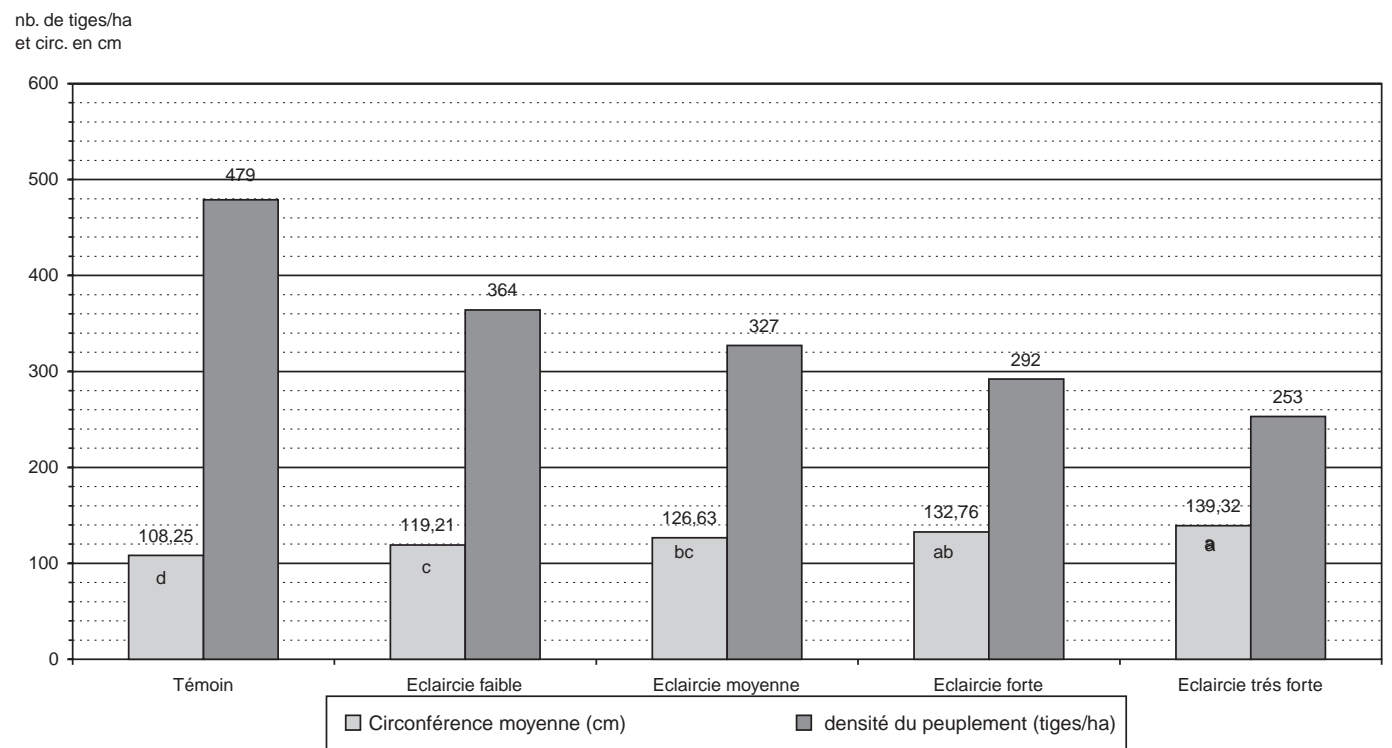

Figure 1. Circonférence moyenne $(\mathrm{cm})$ et densité de peuplement (nombre de tiges/ha), à 50 ans (1998) dans les différentes modalités d'éclaircie. Pour les circonférences, les valeurs affectées de la même lettre ne sont pas statistiquement différentes.

des circonférences les plus élevées, c'est-à-dire d'arbres dominants ou co-dominants.

Au total ce sont 719 carottes qui ont été prélevées et analysées pour cette étude.

\subsection{Mesure des accroissements annuels}

Pour une meilleure visibilité des limites de cerne, chaque carotte de sondage a fait l'objet d'un planage tangentiel à l'aide d'un cutter.

Chez le pin maritime dans les Landes de Gascogne, du fait des vents d'Ouest dominants, il est rare que le cœur de l'arbre soit situé dans l'axe géométrique du tronc. Il y a toujours une certaine « excentricité » du cœur ; anisotropie majoritairement orientée vers le côté « au vent », c'est-à-dire à l'Ouest - Nord-Ouest. De ce fait, malgré les précautions prises, les carottes de sondage atteignent rarement le cœur. Pour faire les corrections nécessaires à la mesure des largeurs de cerne, la distance au cœur est estimée (mesures en $\mathrm{mm}$ ) à l'aide d'une mire circulaire transparente, graduée de 5 en $5 \mathrm{~mm}$. On applique cette mire transparente sur la surface planée de la carotte de manière à faire coïncider la courbure d'un cercle - dont on lit alors le rayon - avec celle du cerne central de la carotte. Plus l'axe de la carotte est excentré, plus cette courbure est faible et plus la longueur du rayon correspondant est grande. De plus, cela permet de mieux apprécier l'âge courant des cernes et de mieux quantifier les surfaces de section.

La mesure des largeurs de cernes est ensuite faite automatiquement, avec une précision du centième de millimètre, sur une image numérique en couleurs, acquise avec un scanner de haute résolution AGFA, à l'aide du logiciel canadien WINDENDRO II (Régent Instrument Inc., Québec).

Le fichier de données ainsi recueillies est ensuite traité avec la série des programmes mis au point par Becker [2] et repris en particulier par Bert [4]. Les phases principales de ce traitement sont classiquement les suivantes.

- Interdatation des courbes individuelles d'accroissement par rapport à une courbe de référence provisoire moyenne calculée et aux «années repères » connues préalablement.

- Transformation des données de largeur de cerne en surface de section.

- Standardisation : pour comparer les surfaces de section (surfaces terrières) des cernes en faisant abstraction de leur âge courant, on calcule des indices de croissance en surface terrière c'est-à-dire, pour chaque arbre et chaque cerne, le pourcentage de la surface terrière développée par rapport à la surface terrière moyenne, à l'âge correspondant, de l'ensemble des arbres témoins. Cette standardisation, qui constitue une étape de la chaîne des traitements, n'était pas vraiment 
indispensable dans notre cas de figure où les arbres ont théoriquement le même âge (futaie régulière).

- Compensation : pour tenir compte d'éventuelles différences de dynamique de croissance entre les placeaux avant l'application des traitements (c'est-à-dire, ici, avant 1967) ; différences qui pourraient être liées à de micro variabilités édaphiques, on calcule, pour chaque traitement, le rapport entre l'indice initial à une date donnée et un indice théorique, à la même date, calculé en utilisant la droite de régression établie sur les 10 valeurs des 10 années antérieures à l'application du traitement. Le nombre de 10 ans est fixé arbitrairement mais correspond à une durée suffisante pour établir une régression solide. Cette opération était nécessaire car, en particulier, la croissance moyenne des arbres dans les placeaux choisis en 1967 comme témoins était avant cette date supérieure à celle des autres.

Les données météorologiques utilisées pour le calcul du bilan hydrique sont celles du poste de BordeauxMérignac distant d'une dizaine de kilomètres de Pierroton. L'ETP a été calculée en utilisant la formule de Thornthwaite [24].

$$
\mathrm{ETP}=16 \times D\left[\left(10 t_{\text {moy }}\right) / l\right]^{a}
$$

où $D$ est un coefficient de correction fonction de la latitude et du mois de l'année, $l$ la somme des $I$ mensuels pour la période de référence, $I$ étant un indice de chaleur et égal à $\left(t_{\text {moy }} / 5\right)^{1,514}$, et $a$ un coefficient dont la valeur approchée est de $0,5+0,016(1)$.

Cette formule présente l'avantage de ne faire appel qu'à des données météorologiques simples. Elle est donc souvent utilisée dans les calculs de bilan hydrique. En particulier, elle a été utilisée par Friend and Hafley [7] dans leur étude sur la limitation climatique de la croissance radiale de Pinus taeda et $P$. echinata.

\section{RÉSULTATS}

\subsection{Dynamique de croissance dans les diverses modalités d'éclaircie}

Les résultats sont exprimés dans la figure 2 montrant l'évolution avec l'âge des moyennes, par traitement, des indices de croissance (définis comme il a été indiqué précédemment). Il s'agit d'indices compensés (cf. infra).

À partir du début de l'expérimentation, la courbe relative aux témoins traduit à la fois l'effet de la compétition

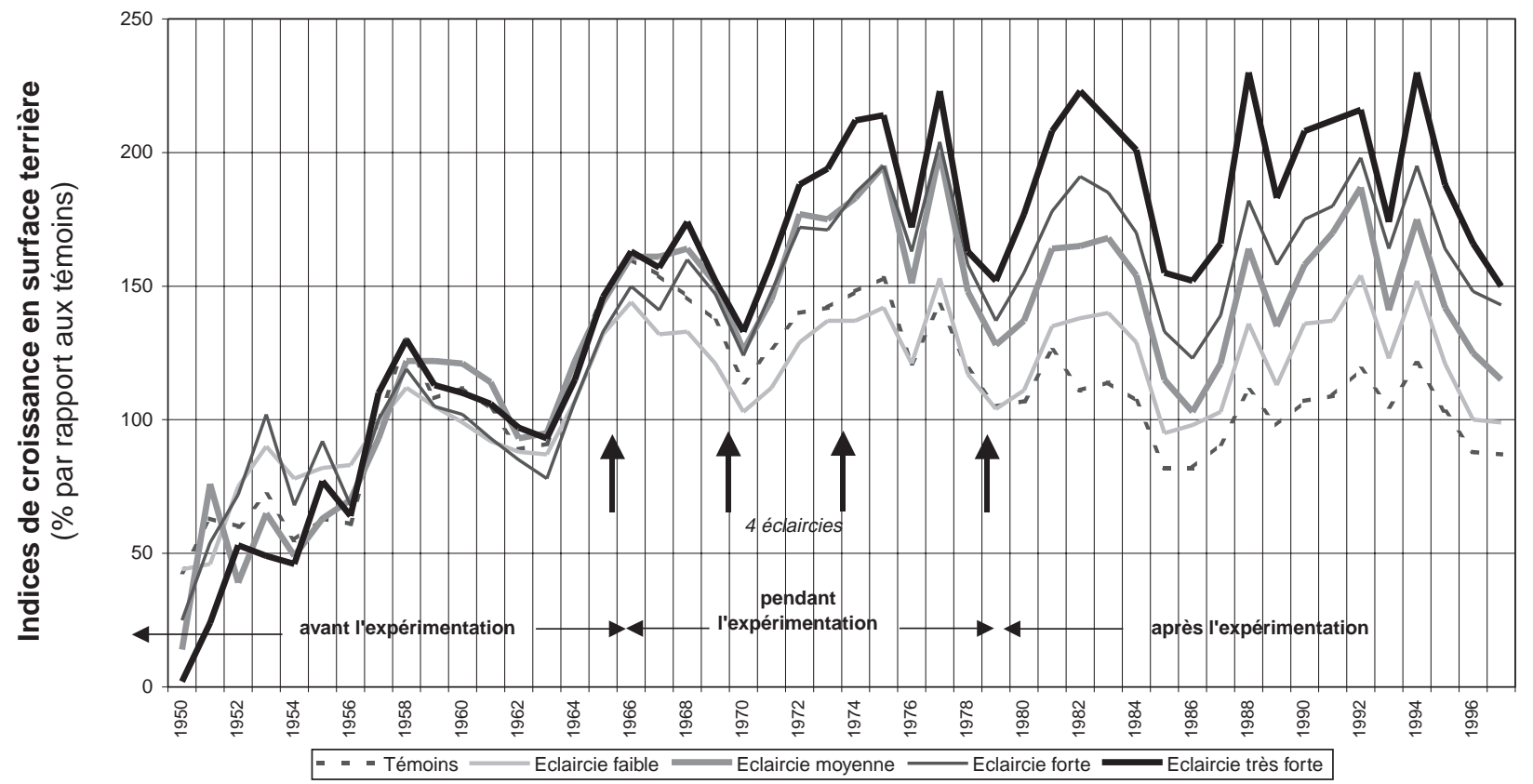

Figure 2. Comparaison de l'évolution, au cours de la vie du peuplement, des indices de croissance en surface terrière dans les 5 modalités d'éclaircie. 


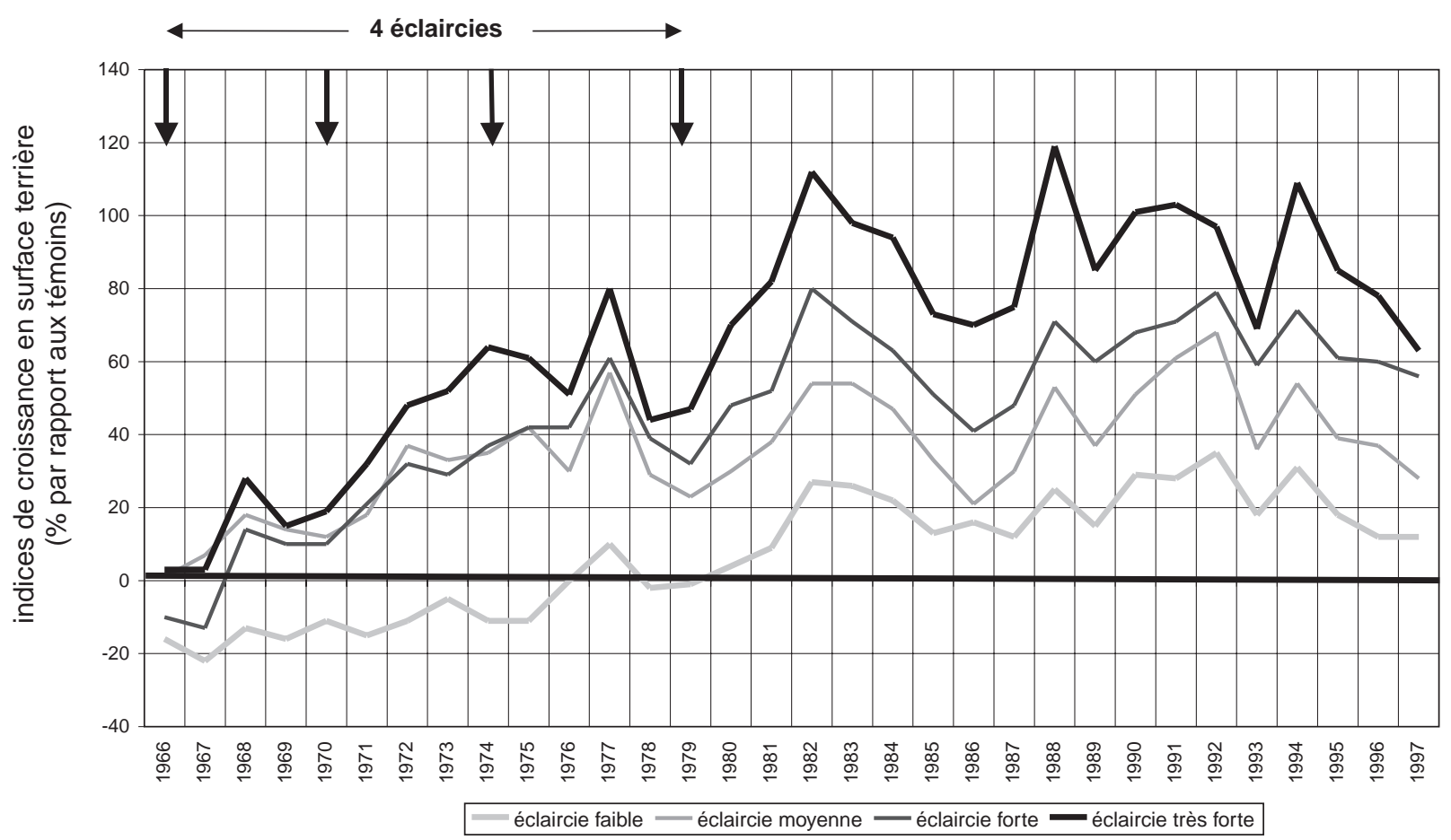

Figure 3. Évolution du gain relatif de croissance en surface terrière dû aux éclaircies.

entre les arbres et l'effet des circonstances climatiques annuelles. La pente de la «droite de tendance » que l'on peut calculer sur la période 1967-1997 $(y=-1,7806 x+$ $147,41)$ peut constituer une estimation et une modélisation de la perte de croissance au cours du temps liée à la concurrence en l'absence d'éclaircies. Les extrema locaux autour de cette moyenne sont à mettre en rapport avec les fluctuations climatiques et, en particulier, avec le bilan hydrique (voir plus loin).

Les minima locaux correspondent aux années 1970, 1976, 1978, (1979), 1985, 1993.

Les maxima locaux correspondent aux années (1975), 1977, (1981), 1988, 1994.

Parmi ces dates, seules celles mises entre-parenthèses ne sont pas significativement différentes, au seuil de $10 \%$, de celles qui l'encadrent. Les autres peuvent donc être considérées comme des années plus ou moins caractéristiques, des « années repères ».

Par rapport à la courbe des témoins, la courbe relative aux éclaircies faibles reflète en plus l'effet des éclaircies, du moins pendant les 12 ans de l'expérimentation; l'écart entre les deux courbes reflétant le gain de croissance dû à ces éclaircies «faibles ». On constate que les
« maxima locaux » et les «minima locaux » de cette courbe coïncident avec ceux de la courbe des témoins et donc aussi avec les fluctuations climatiques. De plus, jusqu'à la dernière éclaircie (1979), la courbe relative à cette modalité « éclaircie faible» se situe «au-dessous » ou au même niveau que celle des témoins, comme si il y avait un effet dépressif de ces éclaircies. En fait, durant cette période, l'écart est le plus souvent non significatif ${ }^{1}$, ce qui prouve que l'éclaircie faible s'est révélée biologiquement trop faible.

Il en est de même pour les courbes relatives aux autres modalités d'éclaircie, à la différence qu'elles se situent toutes, et dans l'ordre du gradient d'intensité d'éclaircie, au-dessus de celle relative aux témoins (figure 2); les écarts étant significatifs.

Le graphique de la figure 3 représente le gain relatif (pourcentage par rapport au témoin) dans les différentes intensités d'éclaircie.

On y voit que, jusqu'en 1982, soit 4 ans après la dernière éclaircie, les différentes courbes sont croissantes ;

1. Les calculs statistiques de significativité sont intégrés aux logiciels de dendroécologie utilisés. 
les gains de croissance allant en augmentant. Il est à noter que les courbes relatives aux éclaircies moyennes et fortes ne se différencient qu'après la $3^{\mathrm{e}}$ éclaircie alors que la courbe relative à l'éclaircie très forte se situe tout de suite à un niveau supérieur aux autres.

Quatre ans après la dernière éclaircie, on atteint un état d'équilibre. Les gains n'augmentent plus, leurs valeurs fluctuant autour d'un plafond en fonction des circonstances climatiques, les écarts acquis précédemment se maintenant.

On peut penser que si une autre éclaircie avait été réalisée à ce moment, on aurait eu un nouveau gain de croissance. Ces résultats justifient une forte périodicité (4 à 5 ans) des éclaircies dans cette période de la vie des arbres et quantifient le gain de croissance obtenu.

Enfin, signalons que la corrélation entre les indices de croissance d'une année $n$ avec ceux de l'année $n+1$, calculée sur la durée de l'expérimentation, est d'autant plus forte que l'intensité d'éclaircie est plus faible (témoins : 0,54 ; intensité moyenne: 0,15 ; intensité très forte: $0,11)$, ce qui tendrait à prouver que dans les fortes éclaircies, il y a une plus grande sensibilité de la croissance aux facteurs externes.

\subsection{Mise en relation avec le bilan hydrique}

La figure 4 donne l'évolution du bilan hydrique $(\mathrm{P}-\mathrm{ETP})$ et de la croissance au cours des années ; ce bilan étant calculé, pour les comparer, de 3 façons : sur l'année entière, sur la période de croissance mars-juillet et la période de croissance mars à octobre. Les décalages que l'on peut constater entre les « maxima locaux » et les « minima locaux » de ces 3 courbes reflètent essentiellement les particularités de la distribution des précipitations des années correspondantes. Dans ce qui suit, nous ne considérerons que les bilans hydriques calculés sur la période de croissance (mars-juillet et/ou mars-octobre).

La mise en relation de cette estimation du bilan hydrique (P - ETP) avec la croissance radiale des pins, ou plutôt des indices de croissance en surface terrière, est donnée par les figures 4,5 et 6 .

Dans la figure 5 on peut comparer l'évolution du bilan hydrique calculé sur les deux périodes de référence définies précédemment par rapport à l'évolution des indices de croissance des seuls témoins ( $\operatorname{str} 1$ ). Nous avons préféré montrer cette comparaison sur un graphique séparé car la figure 4 , où figurent deux autres modalités est de ce fait plus difficile à lire.

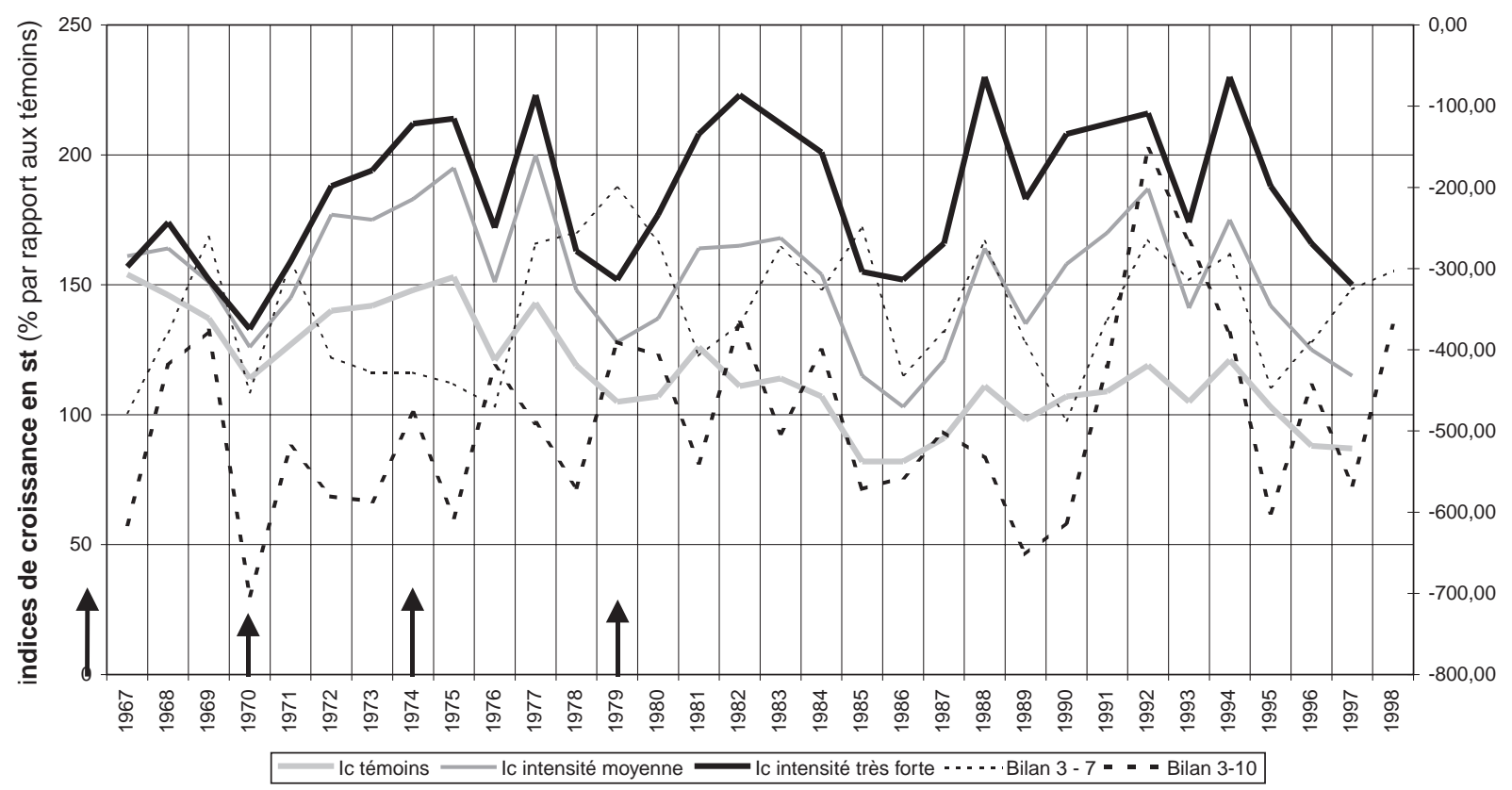

Figure 4. Relation entre le bilan hydrique $(\mathrm{P}-\mathrm{ETP}$, en $\mathrm{mm})$ pour deux périodes de référence et indices de croissance en surface terrière dans 3 modalités d'éclaircie. 


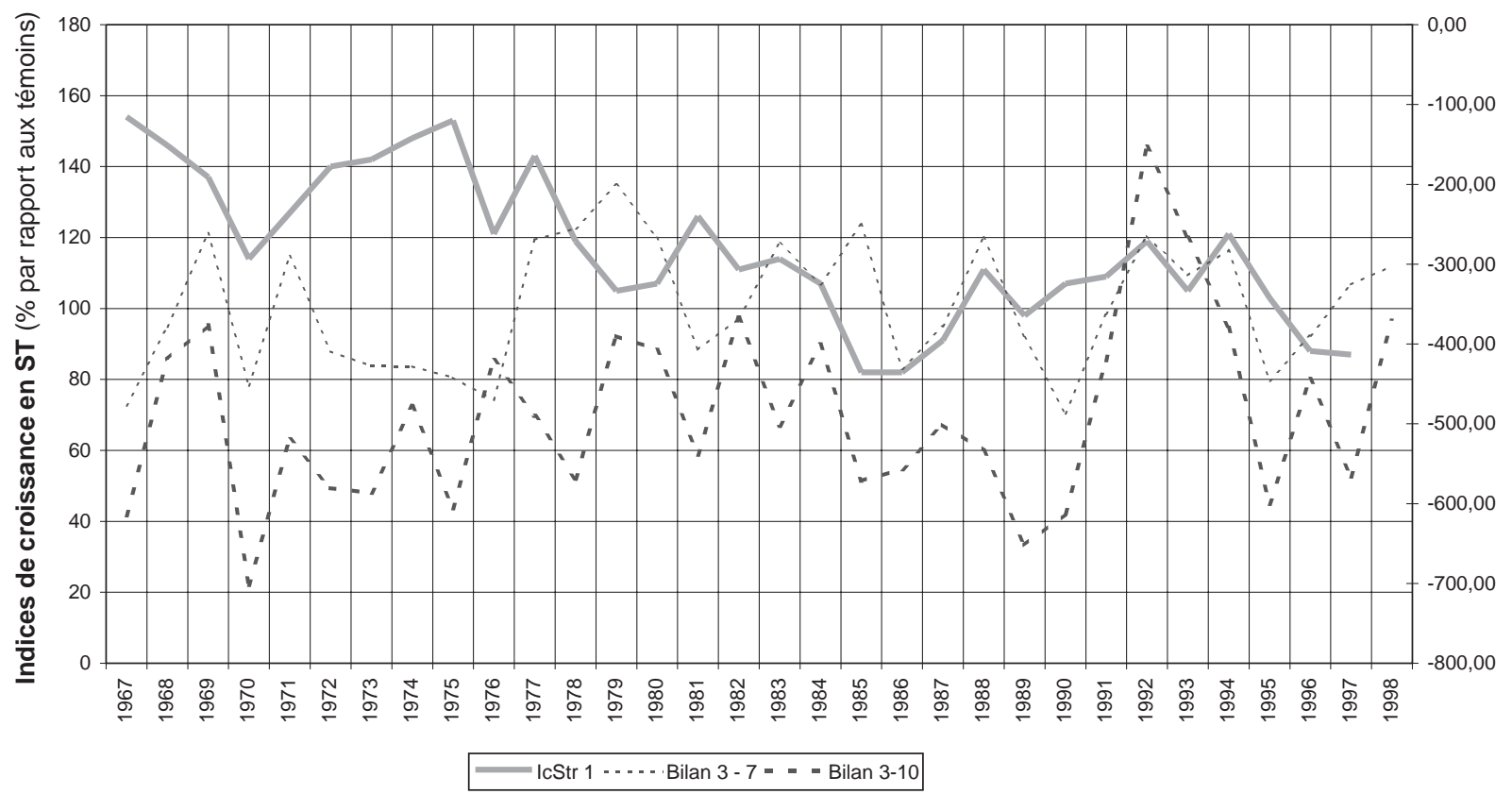

Figure 5. Relation entre le bilan hydrique $(\mathrm{P}$ - ETP, en $\mathrm{mm})$ pour deux périodes de référence et indices de croissance en surface terrière des témoins (IcStr1).

En 1970, la situation est claire : aux «minima locaux » concordant des bilans hydriques, correspond un « minima locaux » de l'indice de croissance des témoins. Il en est de même, mais en sens inverse, pour 1992 : un «pic » dans tous les cas. Dans ces deux cas, l'effet climatique, très fort, masque l'effet de la concurrence. Les autres années l'interprétation est souvent moins aisée; l'effet climatique et l'effet concurrence interférant plus ou moins. De plus on se rend compte que la distribution des pluies au cours de l'année a une influence certaine. Ainsi, par exemple, en 1976, année de sécheresse mémorable, on a effectivement un «minima local» pour la croissance, correspondant bien à un «minima local» pour le bilan hydrique calculé sur la période mars-juillet, mais à un maximum local pour le bilan mars-octobre du fait de précipitations d'arrière-saison (cf. annexe 1). Dans ce cas et dans d'autres (1983 par exemple), le bilan mars-juillet est plus pertinent que le bilan mars-octobre. En 1985, c'est l'inverse ; la correspondance étant meilleure avec le bilan mars-octobre traduisant une sécheresse d'arrière-saison succédant à un printemps et un été «normalement» humide. Certaines années aucune concordance ne peut être mise en évidence, au contraire. Par exemple, en 1979, la croissance est « mauvaise » (un « minima local ») alors qu'on a un «maximum local» pour le bilan hydrique pour les deux périodes de référence. Mais peut-être peut-on y voir un arrière-effet de la sécheresse de la fin de 1978 (conjecturable sur la courbe du bilan mars-octobre). De la même manière, mais en sens inverse, le « bon » bilan hydrique de 1979 et 1980 peut expliquer une «bonne » croissance en 1981 malgré un « mauvais » bilan hydrique cette année-là. Cependant, aucune corrélation significative n'a pu être mise en évidence entre le bilan hydrique d'une année $n$ (ou d'une partie de l'année $n$ ) et la croissance de l'année $n+1$.

On voit là les limites d'un bilan hydrique calculé à partir de la formule de Thornthwaite [24] et donc à partir de données météorologiques simples. On peut penser qu'en particulier un bilan hydrique calculé en tenant compte des réserves hydriques du sol et des fluctuations de la nappe perchée donnerait une meilleure concordance bien que la capacité hydrique des sols sableux landais soit très faible du fait de cette nature sableuse.

Sur la figure 6 on peut voir aussi, en parallèle avec les 2 courbes de bilan hydrique précédentes, les courbes montrant l'évolution de la croissance dans les modalités avec éclaircie. Pour plus de clarté, nous n'avons fait figurer sur ce graphique que la modalité « éclaircie très forte (Str5) » et la modalité médiane (« éclaircie moyenne (Str3)»); les courbes relatives aux deux autres 


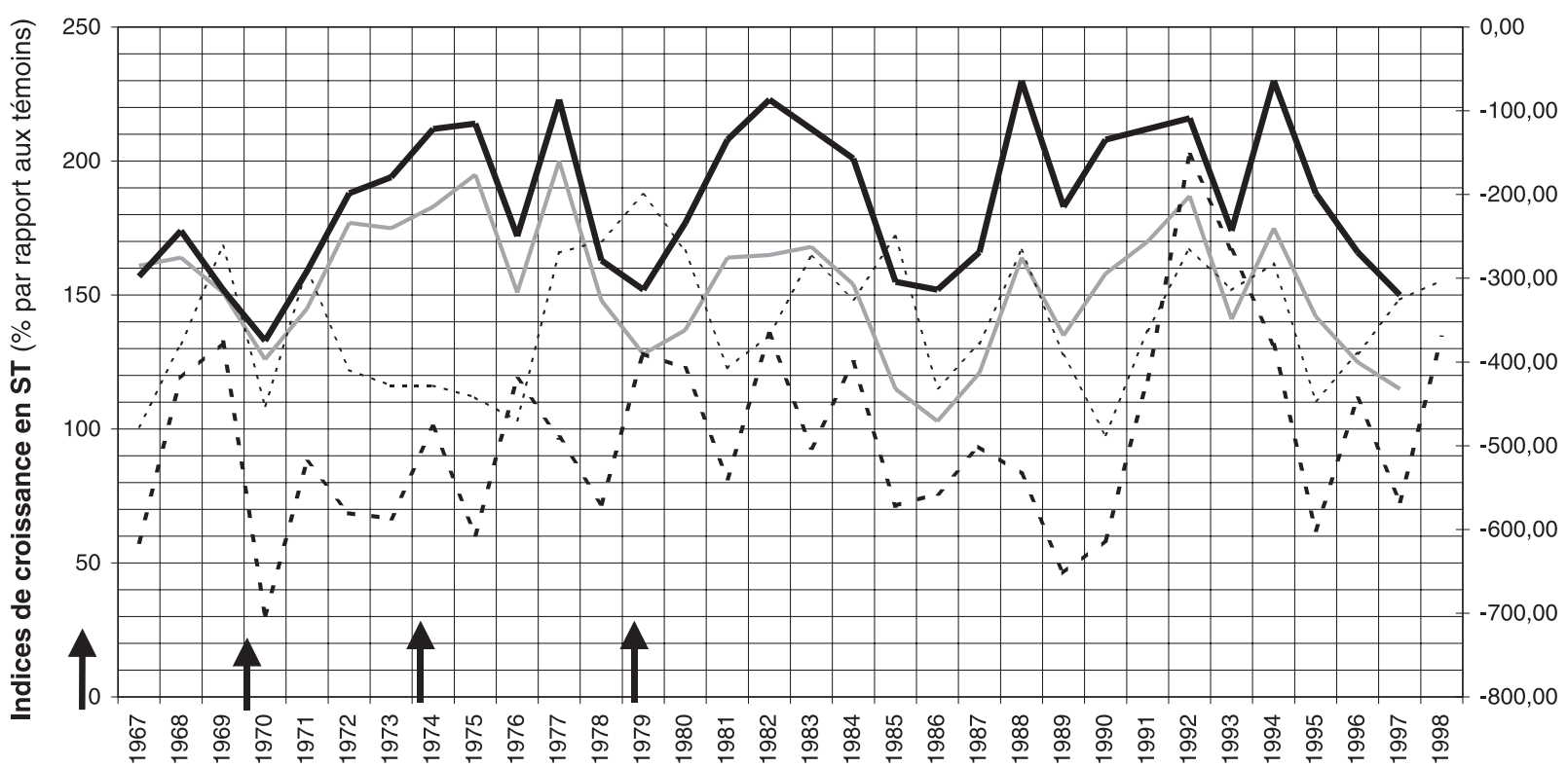

Figure 6. Relation entre le bilan hydrique $(\mathrm{P}-\mathrm{ETP}$, en $\mathrm{mm})$ pour deux périodes de référence et indices de croissance en surface terrière dans 2 modalités d'éclaircie (intensité moyenne, IcStr3 et intensité très forte, IcStr5).

Tableau II. Comparaison des indices de croissance et de leur variation entre 2 des modalités sur les 3 années 1975,1976 et 1977.

\begin{tabular}{|c|c|c|c|c|c|c|}
\hline & & \multicolumn{3}{|c|}{ année } & \multicolumn{2}{|c|}{ Comparaison années } \\
\hline & & 1975 & 1976 (sécheresse) & 1977 & 1976-1975 & 1977-1976 \\
\hline \multirow{3}{*}{ modalités } & témoins & 153 & 121 & 143 & $-20.9 \%$ & $+18 \%$ \\
\hline & Éclaircie moyenne & 195 & 151 & 200 & $-22 \%$ & $+32 \%$ \\
\hline & Éclaircie très forte & 214 & 172 & 223 & $-19,6 \%$ & $+29,6 \%$ \\
\hline \multirow{2}{*}{ Comparaison modalités } & Moyenne - témoin & $+27,4 \%$ & $+24,8 \%$ & $+39,8 \%$ & & \\
\hline & Très forte - témoin & $+39,8 \%$ & $+42,1 \%$ & $+55,9 \%$ & & \\
\hline
\end{tabular}

modalités se situant, d'une manière logique, dans des positions intermédiaires (cf. figure 2).

Analysons ce qui s'est passé de 1975 à 1977 en ayant à l'esprit que cette période fait immédiatement suite à l'éclaircie de 1974 et inclut l'année 1976, fameuse pour sa forte sécheresse (tableau II).

Ce tableau montre plusieurs phénomènes.

- D'abord, quelque soit l'année, l'augmentation des indices de croissance avec l'intensité d'éclaircie (cf. infra) ;
- la pratique des éclaircies, mêmes fortes, n'empêche pas une diminution sensible de la croissance au cours des années de sécheresse (1976 par exemple) où le bilan hydrique est « mauvais »;

- en année de forte sécheresse (1976), avec un « minima local» net pour le bilan hydrique sur la période mars-juillet, on observe une réduction de la croissance qui est du même ordre $(-20 \%)$ dans toutes les modalités ; 
- l'année suivante (1977), avec un bilan hydrique redevenu favorable, on a une augmentation de la croissance du même ordre $(+18 \%)$ que la diminution précédente chez les témoins, alors qu'on a une augmentation supérieure $(+30 \%)$ dans les modalités avec éclaircie ;

- de plus, en 1977, la différence des indices de croissance entre éclaircies et témoin est plus grande qu'en 1975, ce qui peut être considéré comme un effet positif direct de l'éclaircie de 1974.

$\mathrm{Si}$ on examine maintenant la période postérieure à 1979, c'est-à-dire la période postérieure à la dernière éclaircie, on constate que la courbe donnant la valeur des indices de croissance pour les modalités d'éclaircie « moyenne » et « très forte » (figure 6 ), fluctue parallèlement à celle des témoins (figure 3) et parallèlement à celle des bilans hydriques (de mars-juillet et/ou mars-octobre), mais, après chaque « minima local », les « maxima locaux » qui suivent correspondent à des valeurs semblables. Il n'y a donc pas de diminution de la valeur de ces maximums contrairement à ce qui se passe chez les témoins. L'effet de la dernière éclaircie (et des densités de peuplement correspondantes) perdure. Cependant, on constate que plus l'intensité d'éclaircie est forte et plus l'amplitude entre «maxima locaux» et « minima locaux » est forte (tableau III).

Cela tendrait à prouver que dans les modalités fortement éclaircies les arbres sont plus sensibles aux varia- tions inter annuelles du bilan hydrique, dans un sens comme dans l'autre. On peut penser qu'un houppier d'autant plus important que la densité du peuplement est faible est un atout pour la croissance (forte photosynthèse) quand le bilan hydrique est favorable, mais un handicap (forte évaporation) en période de sécheresse.

Une mention particulière doit être faite pour la période 1985-1986 où, pour la période mars-juillet on a un bilan hydrique «favorable » mais « défavorable » pour mars-octobre avec une forte diminution de la croissance dans toutes les modalités. Sachant que le pin maritime est une espèce assez sensible aux basses températures et que des dégâts sont observés lorsque la température descend au-dessous de $-15{ }^{\circ} \mathrm{C}$, on peut y voir les effets des grands froids des hivers de 1985-1986 (annexe II) durant lesquels les cambiums ont souvent été plus ou moins lésés.

Si, sur l'ensemble des années et pour toutes les modalités, on considère le coefficient de corrélation entre les indices d'accroissements en surface terrière et le bilan hydrique calculé sur les 3 périodes de référence évoquées plus haut (tableau II), on constate qu'il est maximum $(0,33$ et 0,25$)$ pour les modalités éclaircies forte et très forte et la période mars-octobre. Pour la modalité témoin, ce coefficient de corrélation est toujours négatif. Pour les modalités « éclaircie forte » et « éclaircie très forte », il est toujours positif et pour les modalités intermédiaires « éclaircie faible » et « éclaircie moyenne » il est positif ou négatif selon la période de référence (tableau IV). Remarquons que les valeurs (positives) de

Tableau III. Amplitudes entre les valeurs « mini » et « maxi » des indices de croissance, après la dernière éclaircie, pour les témoins et deux modalités d'éclaircie.

\begin{tabular}{|c|c|c|c|c|c|c|}
\hline année & 1979 (mini) & & 1982 (maxi) & & 1986 (mini) & 1988 (maxi) \\
\hline \multirow{2}{*}{ Éclaircie très forte } & 152 & & 223 & & 152 & 230 \\
\hline & & $\Delta=71$ & & $\Delta=71$ & & \\
\hline \multirow[t]{2}{*}{ Éclaircie moyenne } & 128 & & 165 & & 103 & 164 \\
\hline & & $\Delta=37$ & & $\Delta=62$ & & \\
\hline témoins & 105 & & 111 & & 82 & 111 \\
\hline
\end{tabular}

Tableau IV. Coefficients de corrélation entre l'indice de croissance en surface terrière et le bilan hydrique pour toutes les modalités et 3 périodes de référence.

\begin{tabular}{|c|c|c|c|c|c|}
\hline $\begin{array}{l}\text { Bilan hydrique } \\
\text { P - ETP }\end{array}$ & $\begin{array}{l}\text { Témoins } \\
\text { str } 1\end{array}$ & $\begin{array}{c}\text { Éclaircies faibles } \\
\text { str } 2\end{array}$ & $\begin{array}{l}\text { Éclaircies moy. } \\
\text { str } 3\end{array}$ & $\begin{array}{l}\text { Éclaircies fortes } \\
\text { str } 4\end{array}$ & $\begin{array}{c}\text { Écl. très fortes } \\
\text { str } 5\end{array}$ \\
\hline Janv. à décembre & $-0,14$ & 0,17 & 0,05 & 0,23 & 0,17 \\
\hline Mars à juillet & $-0,20$ & $-0,03$ & $-0,04$ & 0,07 & 0,03 \\
\hline Mars à octobre & $-0,02$ & 0,30 & 0,20 & 0,33 & 0,25 \\
\hline
\end{tabular}


ce coefficient de corrélation est plus grand dans la modalité « éclaircie forte» que dans la modalité «éclaircie très forte ». On remarquera aussi que quelque soit l'intensité d'éclaircie (mais pas pour lés témoins), ce coefficient de corrélation est maximum pour la période allant de mars à octobre.

\section{CONCLUSIONS}

Plusieurs types de conclusion peuvent être tirés.

D'abord, en ce qui concerne le rythme de la croissance en surface terrière, on constate que, dans la période allant de 1966 à 1979 (de 19 à 32 ans), les 4 éclaircies pratiquées ont permis une croissance des accroissements annuels seulement interrompue par des circonstances climatiques défavorables. Quelques années après la dernière éclaircie, les phénomènes de concurrence entre les arbres font qu'un équilibre s'installe et que les accroissements annuels stagnent en fluctuant au gré des circonstances climatiques. On peut penser que, vu l'âge encore peu élevé du peuplement (32 ans), de nouvelles éclaircies à un rythme analogue auraient permis une prolongation de la période d'accroissements croissants. Cela confirme, le modèle de croissance de Lemoine [14] et précise l'impact de l'effet dépressif des années sèches.

Ensuite, il apparaît que dans le contexte pédologique landais où les sols, sableux, ont une très faible capacité de rétention en eau, les sécheresses peuvent avoir un effet dépressif fort sur la croissance en surface terrière, pouvant aller jusqu'à « gommer » temporairement l'effet bénéfique d'une éclaircie. Plus l'éclaircie est forte plus les arbres sont sensibles aux variations du bilan hydrique, dans un sens comme dans l'autre et plus l'amplitude entre maxi et mini des indices de croissance est importante. Cela est en accord avec les résultats de Misson [19] qui constate que, sur Epicéa, en Wallonie, l'efficacité des éclaircies en cas de sécheresse diminue avec la capacité hydrique des sols. Cet impact négatif des sécheresses se manifeste également dans les différentes intensités d'éclaircie testées, y compris dans la modalité d'éclaircie d'intensité « très forte » où les arbres bénéficient d'une «meilleure » alimentation en eau (concurrence moins grande).

Pour une année donnée, le bilan hydrique, calculé à partir de la formule de Thornthwaite [24] est plus ou moins bien corrélé avec la croissance selon que ce bilan est calculé pour toute l'année ou pour une saison de croissance plus ou moins longue. À ce point de vue, le bilan hydrique calculé sur la période allant de mars à juillet apparaît généralement comme le plus pertinent.
De plus, quelque soit l'intensité d'éclaircie, il y a une faible corrélation entre le bilan hydrique de l'année $n$ et la croissance de l'année $n+1$. Cela est en désaccord avec les résultats de Friend et Hafley [7] qui trouvent un fort effet des conditions hydriques de la période précédant la saison de croissance, mais dans un contexte pédologique très différent (anciens sols agricoles à forte teneur en argile et donc à forte réserve hydrique). De même, contrairement à ces mêmes auteurs, nous ne trouvons pas de corrélations significatives, pour une année donnée, entre les températures printanières et la croissance radiale, quelque soit la modalité d'éclaircie. Il faut noter que Friend and Hafley [7] trouvent certains résultats assez contrastés sur les deux espèces de pin (Pinus taeda et $P i$ nus echinata) étudiés simultanément, ce qui montre que toutes les espèces ne réagissent pas de la même manière.

Cette corrélation de la croissance avec la disponibilité en eau de la saison de croissance est en revanche en accord avec d'autres travaux antérieurs effectués dans ce domaine, mais sur d'autres espèces et dans d'autres contextes pédoclimatiques. En particulier, ils sont en accord avec ceux de Le Goff and Ottorini [13] sur Fagus sylvatica dans le Nord-Est de la France, eux-mêmes en accord avec ceux de Guterriez [9] sur la même espèce en Catalogne espagnole.

Cette sensibilité du pin maritime aux variations annuelles et inter-annuelles du bilan hydrique montre bien son caractère mésophile. Il est probable que l'on aurait obtenu des résultats différents avec une espèce plus xérorésistante ou, toujours avec le pin maritime mais en lande sèche, avec une disponibilité en eau encore plus réduite.

Sur le plan écophysiologique, il apparaît que la réduction de concurrence consécutive à l'éclaircie n'est valorisée sous forme de gain de croissance que si l'alimentation hydrique n'est pas limitante. En effet, en cas de forte sécheresse, et avec des réserves en eau du sol très faibles, les arbres ayant bénéficié de fortes éclaircies et ayant donc développé un important houppier, ont une alimentation hydrique plus difficile et donc une réduction de croissance plus importante.

Sur le plan de la pratique sylvicole, les résultats obtenus confirment et justifient l'intérêt d'un régime soutenu d'éclaircie et plus particulièrement de la périodicité de 4-5 ans à cette époque de la vie du peuplement. où la croissance des pins est active. En revanche, l'intérêt pratique des différentes intensités d'éclaircie doit être relativisé par la production en volume correspondant comme l'ont bien montré Lemoine et Sartolou [17] : «On perd trois fois plus de production quand on passe de l'éclaircie moyenne à l'éclaircie forte que quand on passe de 
l'éclaircie faible à l'éclaircie moyenne », moyennant quoi ils recommandent, comme compromis économique, l'utilisation d'éclaircies d'intensité moyenne. Nos résultats ( $f i$ gure 3) montrant l'absence de différence jusqu'à la troisième éclaircie entre les modalités «éclaircie moyenne » et « éclaircie forte » confirment ce jugement pour cette période.

Remerciements : Je remercie mon collègue Bernard Lemoine (et son technicien A. Sartolou, qui a installé et suivi le dispositif étudié) avec qui nous avons eu des discussions nombreuses et fructueuses sur le sujet. Je remercie également Christophe Gauvrit, technicien de l'Unité expérimentale, qui a effectué le prélèvement des carottes et Fabienne Barrère, stagiaire, qui a effectué une grande partie des mesures de largeur de cerne sur ces carottes. Je remercie enfin Michel Becker pour son aide à la mise en œuvre des programmes de traitement des données et pour sa lecture critique du manuscrit.

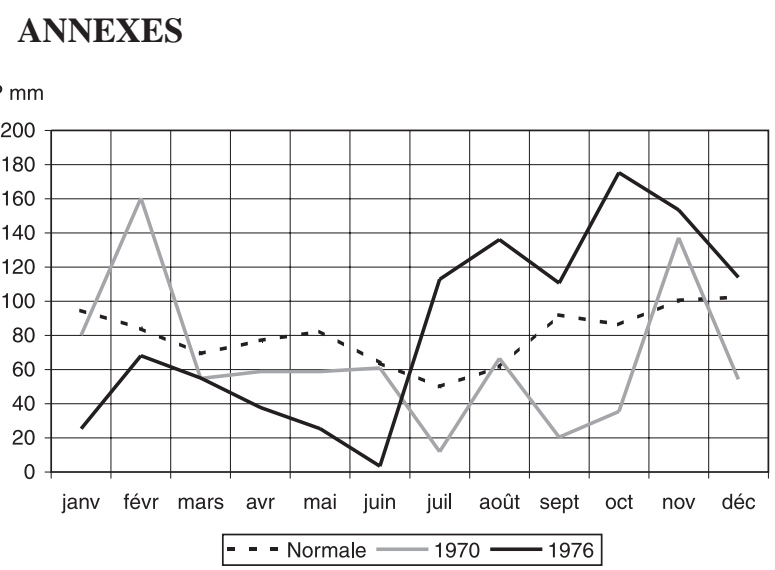

Annexe 1. Mérignac (33), deux années de forte sécheresse: 1970 et 1976.

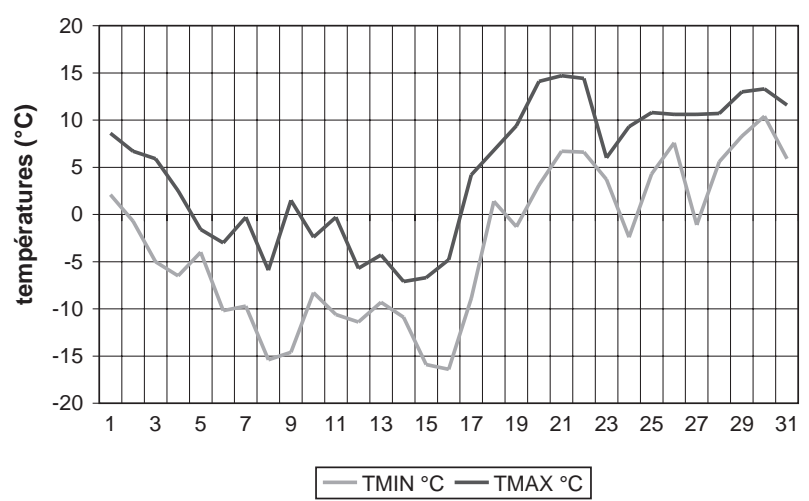

Annexe 2. Mérignac (33), températures de janvier 1985.

\section{RÉFÉRENCES}

[1] Badeau V., Dupouey J.L., Becker M., Picard J.F., Longterm growth trends of Fagus sylvatica L. in north-eastern France. A comparison between high and low density stands, Acta Oecol. 16 (1995) 571-583.

[2] Becker M., The role of climate on present and past vitality of silver fir forests in the Vosges mountains of north-eastern France, Can. J. For. Res. 19 (1989) 110-1117.

[3] Becker M., Radial growth of mature silver firs (Abies alba Miller) fertilized in 1969. Interaction of climate and competition, in: Tree rings and environment. Proceedings of the International Dendrochronological Symposium, Ystad, South-Sweeden, 3-9 September 1990, Lundqua Report. 34, Lund 1992, 17-21.

[4] Bert D., Impact of ecological factors, climatic stress and pollution on growth and health of silver fir (Abies alba Miller) in the Jura mountains: an ecological and dendrochronological study, Acta Oecol. 14 (1993) 229-246.

[5] Cutter B.E., Lowell K.E., Dwyer J.P., Thinning effects on diameter growth in black and scarlet oak as shown by tree ring analysis, For. Ecol. Manage. 43 (1991) 1-13.

[6] Donner B.L., Running S.W., Water stress response after thinning Pinus contorta stands in Montana, For. Sci. 32 (1986) 614-625.

[7] Friend A.L., Hafley W.L., Climatic limitations to growth in Loblolly and Shortleaf Pine (Pinus taeda and P. echinata): A dendrochronological approach, For. Ecol. Manage. 26 (1989) 113-122.

[8] Ginn S.E., Seiler J.R., Cazell B.H., Kreh R.E., Physiological and growth responses of eight-year-old Loblolly pine stands to thinning, For. Sci. 37 (1991) 1030-1040.

[9] Gutierrez E., Dendroecological study of Fagus sylvatica L. in the Montseny mountains (Spain), Acta Ecol. / Ecol. Plant. 9 (1988) 301-309.

[10] Illy G., Lemoine B., Densité de peuplement, concurrence et coopération chez le pin maritime. I. Premiers résultats d'une plantation à espacement variable, Ann. Sci. For. 27 (1970) $127-155$.

[11] Law B.E., Ritters K.H., Ohmann L.F., Growth in relation to canopy light interception in a red pine (Pinus resinosa) thinning study, For. Sci. 38 (1992) 199-202.

[12] Lebourgeois F., Climatic signals in earlywood, latewood and total ring width of Corsican pine from western France, Ann. For. Sci. 57 (2000) 155-164.

[13] Le Goff N., Ottorini J.M., Thinning and climate effects on growth of beech (Fagus sylvatica L.) in experimental stands, For. Ecol. Manage. 62 (1993) 1-14.

[14] Lemoine B., avec la collaboration technique de A. Sartolou, Essai de synthèse biomathématique des aspects concurrentiels, écologiques et cycliques de la croissance du pin maritime dans la pinède des Landes de Gascogne, Ecol. Plant. 10 (1975) 141-167. 
[15] Lemoine B., avec la collaboration technique de A. Sartolou, Pin maritime et sécheresses dans les Landes de Gascogne : croissances en circonférences. C.R, séance du 2 mai 1979 de l'Académie d'Agriculture de France, 694-702.

[16] Lemoine B., Decourt N., Table de production pour le pin maritime dans le Sud-Ouest de la France. Tables de production provisoires, Rev. For. Fr. 26 (1969) 3-44.

[17] Lemoine B., Sartolou A., Les éclaircies dans les peuplements de pin maritime d'âge moyen. Résultats et interprétation d'une expérience, Rev. For. Fr. 38 (1976) 447-457.

[18] Mauge J.P., Le pin maritime, premier résineux de France, IDF, Paris.

[19] Misson L., Approche dendroécologique de l'influence du climat et de l'intensité d'éclaircie sur la croissance radiale de l'épicéa commun (Picea abies (L.) Karsten). Thèse de Doctorat, Faculté des Sciences agronomiques de l'Université catholique de Louvain (Belgique), 2000.
[20] Parde J., Intensité des éclaircies et production ligneuse, Rev. For. Fr. (1964) 936-945.

[21] Parker A.J., Parker K.C., Faust T.D., Fuller M.M., The effects of climatic variability on radial growth of two varieties of Sand pine (Pinus clausa) in Florida, USA, Ann. For. Sci. 58 (2001) 333-350.

[22] Rozas V., Detecting the impact of climate and disturbances on tree-rings of Fagus sylvatica L. and Quercus robur L. in a lowland forest in Cantabria, Northern Spain, Ann. For. Sci. 58 (2001) 237-251.

[23] Splechtna B.E., Dobry J., Klinka K., Tree-ring characteristics of subalpine-fir (Abies lasiocarpa (Hook.) Nutt.) in relation to elevation and climatic fluctuations, Ann. For. Sci. 57 (2000) 89-1000.

[24] Thornthwaite C.W., An approach toward a rational classification of climate, Geogr. Rev. 38 (1948) 55-94.

To access this journal online: www.edpsciences.org 